\title{
Biomimetics Integrated Nanoscience in Dental Tissue Regeneration : A Review
}

${ }^{1}$ Dr. Sonam Gehi, Post graduate student, Department of Prosthodontics, Govt. College of Dentistry, Indore, Madhya Pradesh, India

${ }^{2}$ Dr. Mayank Vagadia, Post graduate student, Department of Prosthodontics, Govt. College of Dentistry, Indore, Madhya Pradesh, India

${ }^{3}$ Dr. Deshraj Jain, Professor and Head, Department of Prosthodontics, Govt. College of Dentistry, Indore, Madhya Pradesh, India

${ }^{4}$ Dr. Alka Gupta, Professor, Department of Prosthodontics, Govt. College of Dentistry, Indore, Madhya Pradesh, India

Correspondence Author: Dr. Sonam Gehi, Post graduate student, Department of Prosthodontics, Govt. College of Dentistry, Indore (M.P.).

\section{Article Info}

Volume 8, Issue 3

Page Number: 289-297

Publication Issue :

May-June-2021

Article History

Accepted : 25 May 2021

Published: 31 May 2021

\begin{abstract}
At a fundamental level, nanotechnology helps to manipulate individual atoms and molecules to produce novel structures with unique properties or improved properties. It involves the production. and applications of physical, chemical, and biological systems and materials at a size scale ranging 1-100 nm. Even though nanotechnology was first introduced over half a century ago, its progress has been slow, but in the last decade, nanotechnology has caught the imagination of scientists and the general public. Nanotechnology offers us the ability to design materials with totally new desirable characteristics Nanotechnology can be approached in two ways: "top-down" and "bottom-up" approaches .Nature uses the bottom-up approach and builds diverse structures in biological systems. The complexity and functionality of these structures is truly amazing. If we can control in fine detail the way in which these structures can be produced in the same way as nature does, remarkable and rapid advances can be made in the field of medicine and dentistry. Nanomaterials will be used far more widely and will yield superior properties and when com bined with biotechnology, laser and digital guided surgery will thus provide excellent dental care. Biomimetics and nanotechnology have given us the knowledge to bioengineer lost tooth and regenerate dental structures. In this review article, recent progress in field of nanotechnology integrated dental tissue regeneration and their potential clinical uses are described.

Keywords:- Nanotechnology, Biomimetics, Bioengineer, Regeneration
\end{abstract}

\section{INTRODUCTION}

Tissue engineering is an interdisciplinary field bringing biology, engineering and clinical science together with aim of reproducing new tissues and organs1. This field of science generates new tissues by combining material science and biocompatibility to integrate cells, natural or synthetic scaffolds and

Copyright: (C) the author(s), publisher and licensee Technoscience Academy. This is an open-access article distributed under the terms of the Creative Commons Attribution Non-Commercial License, which permits unrestricted non-commercial use, distribution, and reproduction in any medium, provided the original work is properly cited 
specific signals. Tissue engineering has also made its path in dentistry in the form of regenerative dentistry. It holds promising future in field of Dental Science. Continuous research is going on at preclinical and clinical level to attain high demand for aesthetics of dental tissue structures and to generate complex atmosphere which dental structures possess2.

Nanotechnology encompasses Science and techniques which manipulate matter at nanometric level. Discovery of Nanotechnology has been widely credited American physicist and Nobel Laureate Dr. Richard Phillip Feymen3.

However, Tanaguchi4 coined the term nanotechnology. It is being researched extensively resulting in generating interesting scientific output and potential commercial applications. There are two approaches to nanotechnology :- the top- down approach encompasses generation of devices via monolithic processing on nanoscale level for construction of semiconductor devices used in electronics. The other bottom up approach involves fabrication of devices via assembling atoms, molecules or basic units of matter used to repair cells tissues and organs systems, by interacting with cell components maniplating cell proliferation and differentiation and organisation of extracellular matrix (ECM). New nanoparticles have led to a range of emerging treatment options that uses biomimetic materials to closely duplicate tooth structure5. In this review article, recent progress in field of nanotechnology integrated dental tissue regeneration and their potential clinical uses are described.

\section{Craniofacial bone and cartilage tissue engineering using nanotechnology}

Conventional strategies to restore stability and function of craniofacial defects secondary to trauma, infection, cancer or congenital disorders is through autografts, allografts or synthetic biomaterials, but they have their own limitations. In this regard, tissue engineering could be considered as a viable approach to repair such defects6. More over combining it with nanotechnology will result in enhanced quality and function of regenerated tissue with optimal physicochemical properties and excellent bio functionalization7.

Various studies within the craniofacial tissue engineering field have revealed successful bone formation with nanofibrous synthetic and natural polymers scaffold such as electrospun polycaprolactone, poly lactic coglycolic acid, polyvinyl alcohol/ Type 1 collagen blend providing optimal environment and cellular platform for bone tissue synthesis [Figure 1] 8,9.

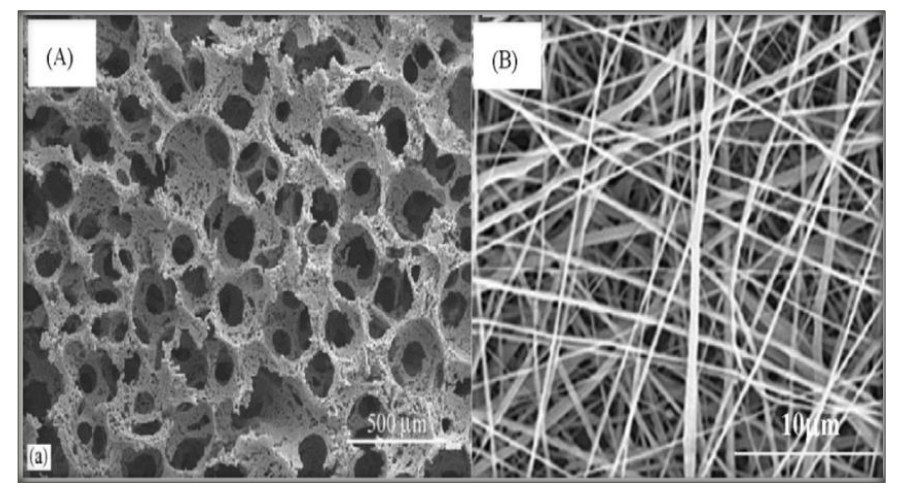

Figure 1 showing (A) Scanning electron microscopy

(SEM) image of poly(L-lactic acid) (PLLA)

nanofibrous scaffold with interconnected spherical macropores created by a phase-separation technique .

(B) Electrospun

polycaprolactone/hydroxyapatite/gelatin

(PCL/HA/gelatin, 1:1:2) nanofibers which significantly improved osteoblast functions for bone tissue engineering applications

Studies done by G. Colon et al and T.J. Webster et al10,11 revealed that nanoceramics have shown increased osteoblast adhesion due to huge surface to volume ratio and unusual chemical synergistic effects such as osteoblast adhesion is increased by $146 \%$ and $200 \%$ on nanophase zinc oxide $(23 \mathrm{~nm})$ and Titanium 
(32nm) comparing to their larger counter parts such as microphase zinc oxide (4.9 micrometre) and Titania (4.1 micrometre) respectively. Therefore, nanomaterials have proved to be better in terms of cell adhesion, proliferation and differentiation which would finally guide tissue regeneration. [figure $2] 12,13$

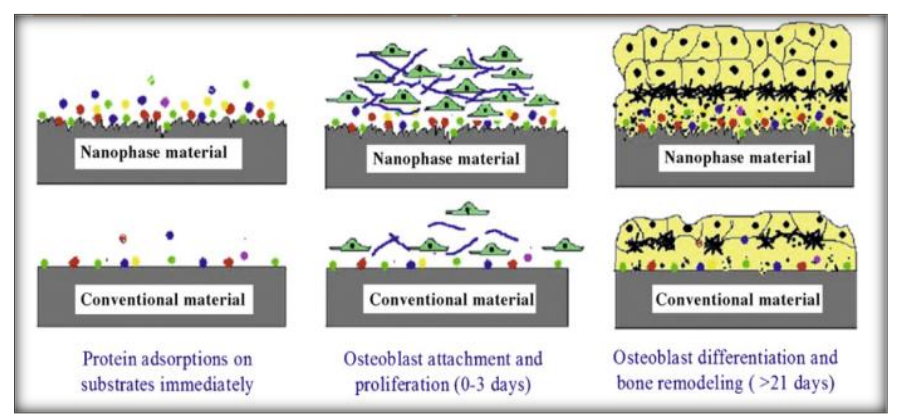

Figure 2 showing schematic illustration of the mechanism by which nanomaterials may be superior to conventional materials for bone regeneration. The bioactive surfaces of nanomaterials mimic those of natural bones to promote greater amounts of protein adsorption and efficiently stimulate more new bone formation than conventional materials.

Nano hydroxyapatite have also proved to be better candidate in terms of bioactivity with improved Cell attachment and mineralization 14 .

First commercial formulation to be accepted by FDA in 2005 used extensively in clinic was NanOss bone from Angstrom Medica Inc. It consists of calcium orthophosphate nanoparticles. Ostim ( Osartis Gmbh and Co. KG, Obernburg, Germany) is another such formula which is a suspension of synthetic nanohydroxyapatite in water used to treat metaphysical fractures, cysts, alveolar ridge augmentation and osteotomies15,16.

Although these inorganic and organic substances have bone regenerating properties but still they have inferior mechanical properties as bone is composed of both organic (collagen Type 1) and inorganic substance (mainly hydroxyapatite), therefore a scaffold should combine both of these components. In this regard, recent studies by Y. Zhang et all7 proposed that chondroitan sulphate in combination with nano-hydroxyapatite showed potential to mimic native bone extracellular matrix to promote bone regeneration. Newer techniques in field of nanotechnology have also lead its path in regeneration of cartilage also which can be helpful in cases of cartilage degradation due to trauma or degenerative articular cartilage destruction of temporomandibular joint with introduction of polymer scaffold having optimal mechanical and degradative properties. Cartilage has limited ability to regenerate due to lack of inductive molecules and vascularity. According to studies done by E. Kon et al18 revealed a biomimetic osteochondral scaffold having nucleating collagen fibrils in combination with hydroxyapatite nanoparticles to repair defects of joints.

Erisken et al19 proposed combination of genetic approach and nanotechnology to direct osteochondral tissue regeneration which could be achieved using nanofibrous scaffold synthesized in two layer containing insulin for chondrogenic differentiation and A- glycerophosphate for osteogenic differentiation.

2. Nanobiomimetic material for periodontal regeneration

Periodontitis often leads to destruction of alveolar bone, cementum, periodontal ligament and gingiva. Conventional treatment modalities for effective periodontal tissue regeneration involve open flap debridement, guided tissue regeneration, bone replacement grafts, but have shown limited and variable clinical outcomes.

Treatment modalities based on concept of endogenous regenerative technology has made its path in periodontics by stimulating latent self repair mechanism of patient and his innate capacity for 
regenerating using patient-derived growth factors, fabrin scaffolds in association with emdogain, bio-Oss to create niche in injury site to be filled with progenitor stem cells from neighbouring tissue and initiate regeneration 20 .

Therefore there is need for some biofunctionalized scaffold mimicking near physiological feature of pore size, interpore connectivity of natural extracellular matrix that incorporate and release regulatory factors facilitating cell recruitment, seeding, adhesion, proliferation, differentiation and neo tissue genesis21. Nano biomimetic materials such as alginate/ nano bioactive glass ceramic composite scaffolds generated using lyophilization having pore size of 400-300 micrometres, controlled porosity, swelling ability, limited degradation, enhanced biomineralization serve as an excellent bioactive matrix for periodontal tissue regeneration without altering viability of MG63 and HPDLF cells and also attained good protein adsorption, cell attachment and proliferation on scaffold showing enhanced alkaline phosphatase activity due to osteoblast like behavior22.Yang et al23 developed a prototype electrospun nanoapatite membrane for guided tissue regeneration showing enhanced bioactivity supporting osteoblast like cell proliferation and differentiation due to presence of nano apatite.

3. Nano technology as a boon for tooth regeneration Tooth regeneration has long been aspiration in dental profession which has come true with combination of tissue engineering and development of genetically designed trigger nano biomimetic particles.

Mao and co-workers, the pioneer researches in dental regeneration, suggested that regeneration of teeth can be divided into several specific areas as follows 24 :-

a) regeneration or de novo formation of an entire, anatomically correct tooth b) regeneration of root

c) regeneration of dental pulp

d) regeneration of dentin that may either act as reparative dentin to seal off an exposed pulp chamber or as a replacement of current synthetic materials

e) regeneration of cementum as a part of periodontium regeneration or for loss of cementum and/or dentin resulting from orthodontic tooth movement

f) regeneration of periodontium including cementum, periodontal ligament and alveolar bone

g) regeneration or synthesis of enamel like structure that may be used as biological substitute for enamel

h) remineralization of enamel and dentin

For tooth regeneration, biomaterials have served as a scaffold primarily for transplanted stem cells or recruitment of endogenous stem cells. The various forms of tooth tissue regenerative engineering related to nanotechnology are described further

A) Nanobiomimetic materials in enamel regeneration Mature enamel being hardest material and highly mineralized skeletal tissue present in body is composed of 95-97\% carbonated hydroxyapatite and less than $1 \%$ organic matter. It has a complex form adapted to absorb mechanical and abrasive stresses throughout life time25. Being acellular and avascular, enamel cannot undergo cellular repair. It loses its mineral content due to various assaults like caries, trauma, erosion and abrasion. Restoration of damage tooth structure is traditional way of treating it but although newer sophisticated restorative materials have been developed but their use still is not completely satisfactory.

Currently, combination of tissue engineering and genetically designed trigger nano bio mimetic particles have begun to show their efficacy in generating teeth tissue. For instance, Amelogenin gene has been genetically transformed which adheres to hydroxyapatite nanoparticles when they are 
directly aimed at pleuripotent cells encased in nano hydrogels and work on formation of enamel tissue. According to research work done by Chen et al26 , synthesized and modified hydroxyapatite nanorod surface with monolayers of surfactants that enabled them to self assemble into prisma like structure at water/ air interface through hydrothermal method, however this method high temperature and pressure and at extremely acidic $\mathrm{pH}$ or concentrated solutions of surfactants. This should be generally avoided as biomimetic material should be generated under physiological environment to be helpful in regenerative process. More recently, $\mathrm{Li}$ et al27 proposed bio-inspired corporative effect of amino acid, glutamic acid and Nano apatite particles to generate enamel like tissue under physiological environment, opening opportunities in near future allowing generation of teeth in dental office.

\section{B) Nanomaterials for enamel and dentin remineralization}

The prevention of tooth decay and the treatment of lesions and cavities are ongoing challenges in dentistry. Currently, biomimetic approaches have been used to develop nanoparticles for remineralization of early enamel lesions28. According to in vitro studies performed by S. Huang et al29, found potential role of nano-hydroxyapatite to remineralize initial enamel carious lesion and the dynamic $\mathrm{pH}$ cycling conditions and its remineralization capacity significantly increases at $\mathrm{pH}$ less than 7. It acts by delivering calcium source to oral cavity, limiting acidic challenge by decreasing enamel demineralization and promoting its remineralization. By acting as calcium phosphate reservoir, it maintains state of supersaturation with respect to enamel minerals therefore decreasing enamel demineralisation promoting its remineralization. It is in accordance to bottom up concept of particle mediated nano precursor assembly and meso crystalline transformation in biomineralization process 30 but only in outer layer of lesion and had limited capacity to reduce lesion depth. L. Li et al31 also demonstrated that evenly sized nanoparticles of 20nm sized hydroxyapatite acted as building blocks of biological appetite of enamel and dentin could more effectively repair and prevent initial lesions in hard tissue of tooth as compared to conventional hydroxyapatite crystals of hundreds nanometres in length. Further studies performed by S. Huang et al32 showed synergistic effect of combining GCE with nano hydroxyapatite in promoting remineralization by producing regularly arranged, dense and uniform crystals in comparison to irregularly arranged crystals while using nano hydroxyapatite alone.

Other biomimetic approaches can be based on nanosized casein phosphopeptide- amorphous calcium phosphate (CPP - ACP) as shown by studies performed by N.J. Cochrane et al33, showed that CPPs stabilize calcium and phosphate ions by forming amorphous nano complexes which enters the porosities at subsurface enamel lesions and after diffusing down the concentration gradient into the body of subsurface lesion releases weakly bound calcium and phosphate ions which fills into crystal voids. Further CPP-ACP also bind to tooth surface and surpragingival plaque to increase bioavailability of calcium and phosphate ions. In an in vitro experiments performed by S. Nakashima et al34, found that nano-sized amorphous calcium carbonate particles applied twice a day for 20 days promoted remineralization of artificial white spot enamel lesions.

Other nano-sized particles promoting enamell remineralization are nano dimensional DCPA based biocomposites having good strength and release of calcium and phosphate ions and nano dimensional beta tricalcium phosphate(TCP)35. Unlike enamel remineralisation, dentine remineralization for treatment of dentinal caries, root caries and dentin hypersensitivity is more challenging because of 
abundance of organic matrix and also dentinal remineralization occurs neither by spontaneous precipitation nor by nucleation of mineral on organic matrix (especially Type 1 collagen) but by growth of residual inorganic crystals in lesions 36.

According to studies done by M. Vollenweider et al37, remineralization of dentin using nano-sized bioactive glass particles and beta- TCP could not reproduce the mechanical properties of original dentin. But as shown by F. R Tray et al38 in their studies, metastable ACP nano precursors generated by the inclusion of polyacrylic acid in phosphate containing fluid, got attracted to acid demineralized collagen matrix and transformed to poly electrolyte stabilized appatite nanocrystals that assemble along microfibrils resulting intrafibrillar remineralisation and surface of collagen fibres resulting interfibrillar remineralization producing remineralized dentin. Results revealed that guided tissue remineralization based on nanotechnology can be useful in remineralization of acid etched dentine which is incompletely infiltrated by dentine adhesives and partially demineralized caries affected dentine.

\section{C) Nanomaterial in dentin-pulp Complex regeneration}

Restorative dentistry is looking for techniques and materials to regenerate dentin-pulp complex in a biological manner. The studies have suggested that odontoblasts ( dentin producing cells), dental pulp stem cells (DPSC) and stem cells from human exfoliated deciduous teeth (SHED) capable of producing pulp/ dentin like tissues when seeded on specific condition or scaffolds39.

Due to the advancements made in the field of biomimetic nanotechnology, various versatile scaffolding materials have been generated which provide satisfactory 3D network to house these cells and direct their growth, organisation and differentiation. In a study done by Rossa et al40 led an important step towards regenerative endodontics, they mixed SHED with nanofiber peptide scaffold and injected it to full-length root canals and were able to generate dental pulp. In another in vitro experiments by K.M. Galler et al41, showed that peptide amphiphile molecules provided nanostructured, cell responsive matrix conducive to dental stem cells. SHED and DPSC seeded in a PA hydrogen showed difference in morphology, proliferation and differentiation behavior. SHED seemed to be useful for soft tissue regeneration whereas DPSC for mineralized tissue like dentin.

\section{CONCLUSION}

Biomimetics and nanotechnology have given us the knowledge to bioengineer lost tooth and remineralize carious lesions. This is one field which has stimulated immense interest among dental and nanotechnology researchers. There has been huge surge in number of studies over the recent few years focusing on clinical applications of Nano biomaterials in dentistry. Despite the challenges in dental tissue regeneration that lie ahead, significant evidence exists to support the premise that recent advances in nanotechnology acting as biomimetic tools, showing great potential to overcome challenges and promises for improved dental tissue regeneration. Nanomaterials tailored for engineering dental tissues are continually being introduced and yield numerous clinical dental benefits. These include improved treatments for periodontal defects, enhanced maxillary and mandibular bone regeneration, perhaps more biological methods to repair teeth after carious damage and possibly even regrowing lost teeth. In the near future, advances in bioengineering research will lead to wide application of regenerative dentistry into general dental practice to produce wonderful treatments and dramatically improve patient's quality of life. 


\section{REFERENCES}

[1]. R. Langer, J.P. Vacanti, Tissue engineering. Science 260 (1993) 920-926.

[2]. S. Yildirim. S.Y. Fu, K. Kim, H. Zhou, C.H. Lee. A. Li. et al., Tooth regeneration: a revolution stomatology and evolution in regenerative medicine, Int. J. Oral Sci. 3 (2011) 107-116.

[3]. R.P. Feynman. There is plenty of room at the bottom. Eng. Sci. 23 (1960) 22-36 and <www.zyvex.com/nanotech/feynman.html/> (1959)

[4]. N. Tanaguchi. On the basic concept of nanotechnology, in: 1974 Proc. ICPE.

[5]. B. Bushan. Springer Handbook of Nanotechnology. 2003, 147-180.

[6]. S.M. Warren, K.D. Fong, C.M. Chen, E.G. Loboa, C.M. Cowan, H.P. Lorenz, et al., Tools and techniques for craniofacial tissue engineering. Tissue Eng. 9 (2003) 187-200.

[7]. L.J. Zhang, T.J. Webster, Nanotechnology and nanomaterials: promises for improved tissue regeneration.Nano Today 4 (2009) 66-80.

[8]. V.J. Chen, P.X. Ma, Biomaterials 25 (2004) 2065.

[9]. J.R. Venugopal, S. Low, A.T. Choon, A.B. Kumar, S. Ramakrishna, Artif. Organs 32 (2008) 388.

[10]. G. Colon, B.C. Ward, TJ. Webster. Increased osteoblast and decreased Staphylococcus epidermidis functions on nanophase $\mathrm{ZnO}$ and TiO2, J. Biomed. Mater. Res. A 78 (2006) 595604.

[11]. T.J. Webster, E.L. Hellenmeyer, R.L. Price, Increased osteoblast functions on theta + delta nanofiber alumina, Biomaterials 26 (2005) 953960.

[12]. I. Degasne, M.F. Baslé, V. Demais, G. Huré, M. Lesourd, B. Grolleau, et al., Calcified Tissue Int. 64 (1999) 499.
[13]. T.J. Webster, C. Ergun, R.H. Doremus, R.W. Siegel, R. Bizios, J. Biomed. Mater. Res. 51 (2000) 475.

[14]. M. Sato, M.A. Sambito, A. Aslani, N.M. Kalkhoran, E.B. Slamovich, TJ. Webster, Increased osteoblast functions on undoped and yttrium-doped nanocrystalline hydroxyapatite coatings on titanium, Biomaterials 27 (2006) 2358-2369.

[15]. R. Smeets, G. Jelitte, M. Heiland, A. Kasaj. M. Grosjean, D. Riediger, et al., Hydroxylapatit Knochenersatzmaterial (Ostim) bei der Sinusbodenelevation, Schweiz Monatsschr. Zahnmed. 118 (2008) 203-208.

[16]. C.K.G. Spies, S. Schnürer, T. Gotterbarm, S. Breusch, The efficacy of Biobon and Ostim within metaphyseal defects using the Göttinger Minipig, Arch. Orthop. Trauma Surg. 129 (2009) 979-988.

[17]. Y. Zhang, v.J. Reddy, S.Y. Wong, X. Li, B. Su, S. Ramakrishna, et al. Enhanced biomineralisation in osteoblasts on novel electrospun biocomposite nanofibrous substrate of hydroxyapatite/collagen/ chitosan, Tissue Eng Part A 16 (2010) 1949-1960

[18]. E. Kon. M. Delcogliano, G. Filardo, D. Pressato, M. Busacca, B. Grigolo, et al., A novel nanocomposite multilayered biomaterial for treatment of osteochondral lesions: technique note and an early stability pilot clinical trial. Injury 41 (2010) 693-701

[19]. C. Erisken. D.M. Kalyon. H. Wang, C. OrnekBallanco, 1. Xu, Osteochondral tissue formation through adipose derived stromal cell differentiation on biomimetic polycaprolactone nanofibrous scaffolds with graded insulin and Beta-glycerophosphate concentrations, Tissue Eng. Part A 17 (2011) 1239-1252.

[20]. F.M. Chen, J. Zhang, M. Zhang, Y. An, F. Chen, $\mathrm{ZF}$. Wu. A review on endogenous regenerative technology in periodontal regenerative medicine, Biomaterials 31 (2010) 7892-7927. 
[21]. P.X. Ma, Biomimetic materials for tissue engineering. Adv. Drug. Deliv. Rev. 60 (2008) 184-198.

[22]. S. Srinivasan, R. Jayasree, K.P. Chennazhi. S.V. Nair, R. Jayakumar, Biocompatible alginate/nano bio active glass ceramic composite scaffolds for periodontal tissue regeneration, Carbohydr. Polym. 87 (2012) 274-283.

[23]. F. Yang. S.K. Both, X. Yang, X.F Walboomers. JA. Jansen, Development of an electrospun nano-apatite/ PCL composite membrane for GTR/GBR application. Acta Biomater. 5 (9) (2009) 3295-3304

[24]. Z. Yuan, H. Nie. S. Wang, C.H. Lee, A. Li. S.Y. $\mathrm{Fu}$, et al., Biomaterial selection for tooth regeneration. Tissue Eng. Part B Rev. 17 (2011) 373-388.

[25]. A.G. Fincham. J. Moradian-Oldak, J.P. Simmer. The structural biology of the developing dental enamel matrix. J. Struct. Biol. 126 (1999) 270299

[26]. H.F. Chen, B.H. Clarkson, K. Sun, J.F. Mansfield, Self-assembly of synthetic hydroxyaptite nanorods into enamel prism like structure. J. Colloid Interface Sci. 188 (2005) 97103

[27]. L Li, C. Mao, J. Wang, X. Xu, H. Pan, Y. Deng, et al., Bio-inspired enamel repair via Giudirected assembly of apatite nanoparticle: an approach to biomaterials with optimal characteristic, Adv. Mater. 23 (2011),4695-4701.

[28]. M. Hannig, C. Hannig, Nanomaterials in preventive dentistry. Nat. Nanotechnol. 5 (2010) 565-569

[29]. S. Huang. S. Gao, L. Cheng, H. Yu, Remineralization potential of nanohydroxyapatite on initial enamel lesions: an in vitro study, Caries Res, 45 (2011) 460-468.

[30]. A.W. Xu, Y.R. Ma, H. Colfen, Biomimetic mineralization, J. Mater. Chem. 17 (2007) 415449.
[31]. L. Li, H.H. Pan, J.H. Tao, X.R. Xu, C.Y. Mao, X.H. Gu, et al., Repair of enamel by using hydroxyapatite nanoparticles as the building blocks, J. Mater. Chem. 18 (2008) 4079-4084.

[32]. S. Huang, S. Guo, L. Cheng, H. Yu, Combined effects of nano-hydroxyapatite and Galla chinensis remineralization of initial enamel lesion in vitro, J. Dent. 38 (2010) 811-819.

[33]. NJ. Cochrane, F. Cai, N.L. Huq. M.F. Burrow, E.C. Reynolds, New approaches to enhanced reminerali zation of tooth enamel, J. Dent. Res. 89 (2010) 1187-1197.

[34]. S. Nakashima, M. Yoshie, H. Sano, A. Bahar, Effect of a test dentifrice containing nano-sized calcium carbonate on remineralization of enamel lesions in vitro, J. Oral Sci. 51 (2009) 6977 .

[35]. Y.W. Hong, J.H. Kim, B.H. Lee. Y.K. Lee, B.J. Choi, J.H. Lee, et al., The effect of nano-sized 3tricalcium phosphate on remineralization in glass ionomer dental luting cement. Key Eng. Mater. 361-363 (2008) 861-864

[36]. K. Kawasaki, J. Ruben, I. Stokroos, O. Takagi, J. Arends., The remineralization of EDTA-treated human dentine, Caries Res. 33 (1999) 275-280.

[37]. M. Vollenweider, TJ. Brunner, S. Knecht, R.N. Grass, M. Zehnder, T. Imfeld, et al., Remineralization of human dentin using ultrafine bioactive glass particles, Acta Biomater. 3 (2007) 936-943.

[38]. F.R. Tay, D.H. Pashley, Biomimetic remineralization of resin-bonded acid-etched dentin, J. Dent. Res. 88 (2009) 719-724

[39]. V.T. Sakai. Z. Zhang, Z. Dong. K.G. Neiva, M. Machado, S. Shi, et al., SHED differentiate into functional odontoblast and endothelium, J. Dent. Res. 89 (2010) 791-796.

[40]. V. Rosa. A. Della Bona, B.N. Cavalcanti, J.E. Nör, Tissue engineering: from research to dental clinics, Dent. Mater. 28 (2012) 341-348.

[41]. K.M. Galler, A. Cavender, V. Yuwono, H. Dong, S. Shi. G. Schmalz, et al., Self-assembling 
peptide amphiphile nanofibers as a scaffold for dental stem cells, Tissue Eng. Part A 14 (2008) 2051-2058.

\section{Cite this article as :}

Dr. Sonam Gehi, Dr. Mayank Vagadia, Dr. Deshraj Jain, Dr. Alka Gupta, "Biomimetics Integrated Nanoscience in Dental Tissue Regeneration : A Review", International Journal of Scientific Research in Science, Engineering and Technology (IJSRSET), Online ISSN : 2394-4099, Print ISSN : 2395-1990, Volume 8 Issue 3, pp. 289-297, May-June 2021. Available

doi : https://doi.org/10.32628/IJSRSET218344

Journal URL : https://ijsrset.com/IJSRSET218344 